\title{
Strates
}

STRATES Matériaux pour la recherche en sciences sociales

13 | 2007

Paysage urbain: genèse, représentations, enjeux contemporains

\section{Les villes nouvelles françaises : lieux de formation aux pratiques de l'aménagement}

\section{Viviane CLAUDE}

\section{(2) OpenEdition}

\section{Journals}

Édition électronique

URL : http://journals.openedition.org/strates/5853

DOI : $10.4000 /$ strates.5853

ISSN : $1777-5442$

Éditeur

Laboratoire Ladyss

Édition imprimée

Date de publication : 31 décembre 2007

ISSN : 0768-8067

Référence électronique

Viviane CLAUDE, «Les villes nouvelles françaises : lieux de formation aux pratiques de

l'aménagement », Strates [En ligne], 13 | 2007, mis en ligne le 22 octobre 2008, consulté le 08

septembre 2020. URL : http://journals.openedition.org/strates/5853 ; DOI : https://doi.org/10.4000/

strates.5853

Ce document a été généré automatiquement le 8 septembre 2020

Tous droits réservés 


\title{
Les villes nouvelles françaises : lieux de formation aux pratiques de l'aménagement
}

\author{
Viviane CLAUDE
}

1 La décision de construire des villes nouvelles s'est accompagnée en France de la création d'organismes éphémères et singuliers : d'abord des « Missions » puis, de façon plus officielle après les premiers décrets d'avril 1969, des Établissements publics d'aménagement des villes nouvelles (EPAVN) qui ont aujourd'hui pour la plupart disparu. Ces organismes avaient vocation à mener de façon enchaînée un ensemble de tâches, des études générales d'aménagement jusqu'à la remise d'ouvrages aux Syndicats communautaires d'agglomération, devenus après 1983 Syndicats d'agglomération nouvelle. Dans cette production intégrée et qui allait jusqu'à l'accueil des premiers habitants, des «métiers » nouveaux sont apparus. C'est notamment le cas dans le domaine de la programmation (à la fois la programmation urbaine générale et celle des équipements publics) et dans celui du traitement du paysage à ses différentes échelles, des espaces publics à l'aménagement de grands territoires. Ces nouveaux métiers viennent étoffer un milieu professionnel devenu généralement plus bigarré, suite à l'amplification et à la diversification de la commande publique en matière d'aménagement au cours des années $1960^{1}$.

2 Les EPAVN sont loin d'avoir suivi la même trajectoire. Ils se transforment de façon relativement indépendante les uns des autres, du fait des enjeux sociopolitiques locaux et des caractéristiques du territoire sur lequel se construit la ville nouvelle. Tout sépare par exemple l'Étang-de-Berre de Lille-Est. Ils diffèrent par ailleurs dans leur organisation interne, même si une tendance générale se dégage : à un modèle plutôt vertical, hiérarchique et par spécialité, succède un modèle davantage horizontal, par secteur territorial ou par projet. Les EPAVN ont aussi exercé plusieurs fonctions fonction d'études, fonction opérationnelle, fonction commerciale et, au-delà, remise d'ouvrage - qui s'articulent différemment selon le moment et l'établissement. Enfin, entre architectes, ingénieurs, paysagistes, spécialistes des sciences humaines, juristes, 
gestionnaires..., les relations professionnelles sont loin d'avoir obéi à un schéma identique, d'avoir connu les mêmes ressorts et d'avoir produit les mêmes effets. Dans tous les EPAVN, elles ont cependant été marquées par le travail en équipe et par ce que l'on a appelé la pluridisciplinarité ${ }^{\text {. }}$

Ces activités nouvelles se révèlent en même temps que les premières équipes des EPAVN se forment et ceci dans les deux sens du mot: les équipes se constituent et apprennent à travailler ensemble dans un domaine, l'aménagement, auquel elles sont pour la plupart peu préparées, vu leur jeune âge et la volonté générale "d'innover » à un moment où les réalisations françaises déçoivent. Il s'agit, dans la sphère administrative comme dans les milieux professionnels, de rompre avec la "banlieue » et les "grands ensembles", de "faire de la vraie ville ${ }^{3}$ ». Les conditions matérielles, institutionnelles et idéologiques sont réunies pour que, à partir des pratiques concrètes des professionnels, se dessine le « champ » de l'aménagement, comme ensemble plus ou moins unifié de savoirs et savoir-faire. Ce sont moins des "professions » nouvelles qui vont apparaître que des pratiques qui s'élargissent, des expertises qui s'instituent et des collaborations qui s'expérimentent ${ }^{4}$. Car les expériences individuelles et collectives au sein des EPAVN débordent de beaucoup les compétences reconnues aux professions stabilisées voire protégées. Ces expériences illustrent pour cette période des années 1960 et au-delà, deux caractéristiques liées au milieu professionnel de l'aménagement urbain, d'abord son caractère fragmenté ensuite l'enjeu que constitue l'organisation des coopérations ${ }^{5}$.

4 À la constitution de ce champ, les paysagistes, dont l'histoire est de mieux en mieux connue $^{6}$, ont apporté une triple contribution. D'abord en prenant place dans la maîtrise d'ouvrage, ce à quoi leur formation tout comme celle des architectes ou des ingénieurs ne les prépare pas. Ensuite, en travaillant, de la même manière que les autres, sur des questions et thèmes encore peu explorés. Enfin et par la suite en insérant leurs façons de voir et de faire dans les pratiques collectives. Dans des situations de collaboration étroite, ils ont trouvé l'occasion d'étendre leurs compétences, de faire entendre leur voix tout en s'affranchissant des professions plus affirmées (architectes et ingénieurs) mais qui n'étaient pas plus familières qu'eux des questions d'aménagement dans les années 1960. De façon générale, les expériences acquises en villes nouvelles ont enrichi la culture de l'aménagement ${ }^{7}$. Ce n'est là qu'une hypothèse de travail compte tenu des ressources disponibles: les pages qui suivent s'appuient pour beaucoup sur des témoignages et la mémoire faillible des acteurs. Sur quelques indices précis, on peut cependant identifier la part qui reviendrait en propre aux savoirs et aux savoir-faire des paysagistes dans cet enrichissement, autrement dit leur rôle " d'expert instituant " qui déborde de leur capacité reconnue à la gestion du milieu végétal ${ }^{8}$.

Les contextes

$5 \quad$ Les conditions de naissance des villes nouvelles sont assez largement connues' ${ }^{9}$. Il faut ici rappeler quelques éléments du contexte particulièrement favorable dans lequel les professionnels vont travailler. La volonté politique qui soutient initialement la construction des villes nouvelles s'accompagne de moyens importants: création de Zones d'aménagement différé sur de vastes territoires, permettant de contrôler le marché foncier, de lever l'obstacle de la propriété foncière, de mener des réflexions à l'échelle de grands sites; mise en place de cellules d'études (les Missions, structures légères et relativement autonomes, qui précèdent la création des EPAVN); appel à des compétences variées, non seulement par les qualifications initiales mais aussi par le 
passé professionnel des agents recrutés ${ }^{10}$. À cet égard, il ne faut pas s'étonner qu'une expérience coloniale (" savoir faire avec rien ») ait été souvent appréciée ${ }^{11}$.

Dans les premières Missions, ce sont surtout des architectes, ingénieurs, administrateurs et quelques rares diplômés en sciences humaines qui sont recrutés. Ils doivent alors tout inventer et sont pris dans un bouillonnement d'idées et dans des discussions dont les anciens gardent le souvenir ${ }^{12}$. Le travail de ces équipes initiales est tiré par des idéaux politiques et par les grands projets qu'inspirent des territoires considérés comme vierges. Les perspectives sont alors fort larges, comme en témoigne aujourd'hui un paysagiste à l'Étang-de-Berre ${ }^{13}$ :

Nos prévisions étaient alignées sur un haut-fourneau, deux hauts-fourneaux, quatre hauts-fourneaux... Elles étaient alignées comme ça. Elles allaient droit sur la lune. Ce délire, il était fait sur une courbe de croissance d'avant le choc pétrolier et c'était une pure fiction d'ingénieurs.

Dans la période 1973-1980, la conjoncture est différente. D'abord, les premiers effets de la crise économique se font sentir. À l'Étang-de-Berre, ce fut immédiat. À Cergy, la proximité de La Défense fait craindre une concurrence plus vive dans la mise sur le marché de bureaux. Or, dans ces années 1970, les EPAVN sont dans un processus irréversible de production urbaine : des infrastructures, des milliers de logements, des écoles sortent de terre et les premiers habitants s'installent. Après les élections municipales de 1977, les structures syndicales (Syndicat communautaire d'agglomération puis Syndicat d'agglomération nouvelle) s'étoffent en personnel. Les professionnels des EPAVN ne sont plus dans un monde où les arbitrages se faisaient entre eux ${ }^{14}$. Dans certains cas, à Lille-Est par exemple, les élus manifestent le souci de mieux maîtriser voire de freiner la réalisation des équipements : « Les élus trouvaient qu'il y avait trop de maisons de quartier, trop d'espaces verts ${ }^{15}$. » Les approches se font alors plus pragmatiques, tant vis-à-vis des élus que vis-à-vis des habitants, la « sanction des usages effectifs » obligeant à davantage d'attention aux processus d'appropriation des espaces produits. Le sociologue Jean Rémy livre à l'EPAVN de Lille-Est son expérience de Louvain-la-Neuve et accompagne ce pragmatisme critique ${ }^{16}$. Dans cette conjoncture, les approches sont aussi plus soucieuses des réalités économiques, ce que rappelle le paysagiste de l'Étang-de-Berre ${ }^{17}$ :

On était innocent à l'époque, on ne s'intéressait pas au marché ; ça a entretenu des illusions par ce qu'on n'a pas mesuré assez vite dans quelle compétition commerciale, mais pas seulement commerciale, médiatique, idéologique on était avec des villes comme Aix.

8 Les années 1980 apportent leur lot de transformations. Avec la décentralisation, il a fallu préparer l'entrée des villes nouvelles dans le droit commun. La loi du 13 juillet 1983, dite loi Rocard, modifie le «statut des agglomérations nouvelles » en donnant aux collectivités locales une plus large maîtrise de leurs équipements et des ressources fiscales nécessaires (taxe professionnelle unique) ${ }^{18}$. Puis viennent les premiers effets de la réforme du logement de 1977: la composition sociodémographique de ces agglomérations se remodèle et les premières poches de paupérisation apparaissent ${ }^{19}$. Enfin, la disparition des EPAVN est à l'horizon voire effective. Celui de Lille-Est, ferme ses portes, le premier, en 1985. Ailleurs, dans la foulée, la vente des charges foncières est accélérée.

Les organisations

9 Sur trois décennies, les objectifs et les conditions du travail dans les Missions et EPAVN se sont largement modifiés et avec eux les formes de la fameuse pluridisciplinarité, 
terme dont le succès est à la hauteur du flou qui entoure son sens ${ }^{20}$. Ce que vivent au quotidien les professionnels, ce sont leurs interdépendances. Leur travail, les relations comme les divisions qu'il provoque, dépend des formes d'organisation adoptées, de leur transformation au cours du temps, et en même temps de la mobilisation des compétences extérieures.

10 Les professions constituées pèsent de tout leur poids dans les effectifs des EPAVN : architectes et ingénieurs « règnent » sur les services à la fois dans la hiérarchie et sur tout le processus de production, dans la phase des études comme dans la phase opérationnelle. Ils occupent sur toute la période (1965-2000) $33 \%$ des postes de cadres à Evry et Cergy, $49 \%$ à l'Étang-de-Berre, avec des variations importantes selon les structures et les périodes ( $45 \%$ d'architectes à Evry en 1970, 42 \% d'ingénieurs à Cergy en 1976) ${ }^{21}$. Ceux que l'on désigne comme les "architectes-urbanistes" ont été particulièrement puissants à Cergy ou Evry où ils ont tenu des rôles "d'urbanistes en chef ». Ainsi l'atelier d'urbanisme de la ville nouvelle d'Evry est apparu comme un «État dans l'État » qui réunit des architectes et des programmateurs. En revanche à Marne-la-Vallée, les architectes-urbanistes n'ont pas eu une telle place, ayant par exemple moins de prise sur le choix des maîtres d'œuvre: "Pour les dossiers de consultation, les urbanistes n'étaient que consultés; ils ne les produisaient pas ${ }^{22}$. »

11 Les paysagistes, quant à eux, n'ont pas été très nombreux : sur trois des villes nouvelles citées (Cergy, Evry, Étang-de-Berre), ils ont été au total neuf à y faire un bout de leur carrière entre 1967-1968 et 2000 (quatre d'entre eux sont diplômés de l'École nationale supérieure du paysage). En moyenne, on compte un paysagiste pour sept à huit architectes. De même que les programmateurs, les paysagistes ont dû faire leur place. Leur position variable dans les organigrammes - leur dépendance à l'égard des professions constituées - décide des collaborations qui se sont nouées. Il y a des différences en effet entre ceux qui ont travaillé avec les architectes et ceux qui ont été aux côtés des ingénieurs. Par leur faiblesse numérique, ils n'ont jamais pu être en concurrence avec les uns ou avec les autres. Ils ont été plutôt enrôlés dans des partenariats variés qui leur ouvrent plus ou moins de possibilités d'être reconnus comme spécialistes. Ces coopérations conditionnent le sens, l'orientation et la signification du métier.

12 Selon les organigrammes et selon la présence ou non d'un "urbaniste en chef ", l'étendue et la nature des activités des paysagistes sont donc assez différentes. À Marne-la-Vallée, ils ont été associés aux ingénieurs dans un service opérationnel pour assurer au mieux le lien entre conception et réalisation. À l'Étang-de-Berre, le paysagiste assure la coordination de la production architecturale, exerçant ainsi des compétences qui sont ailleurs réservées aux architectes. Forts de leur position de maître d'ouvrage, ils mettent en cause les normes et les manières de faire ordinaires. L'un d'entre eux se souvient ${ }^{23}:$ «Je passais mon temps à faire en sorte que les ratios consacrés à l'espace public ne soient pas des ratios mais soient des projets. » Plus leur présence est forte au sein des EPAVN, plus est systématique le recours à des maîtres d'œuvre paysagistes. C'est le cas à Marne-la-Vallée et à l'Étang de Berre.

13 Force de propositions, prenant parfois des positions critiques, les bureaux d'études extérieurs ont de manière générale contribué à la professionnalisation de la maîtrise d'ouvrage, en même temps qu'ils étendaient leurs compétences ou se formaient à d'autres pratiques ${ }^{24}$. Ainsi les paysagistes maîtres d'œuvre, non contents de leur rôle d'« habilleur ", ont bousculé la division traditionnelle du travail. Pour échapper aux 
façons de faire antérieures où « le domaine du paysagiste était tout ce qui était planté, tout ce qui était vert et ce qui était structurant était le domaine de l'ingénieur ", l'un de ces maîtres d'œuvre négocie chaque terme des contrats d'études ${ }^{25}$. Sa position de prestataire comme ce qu'il défend comme étant son métier lui offre des libertés nouvelles ${ }^{26}$ :

On n'était pas un corps constitué, on n'était pas issu d'une technicité reconnue, qui avait son école... Il n'y avait pas ce côté corporatif comme chez les architectes, les ingénieurs... Nous on se baladait entre tout ça, et on a pris pied là où personne ne voulait aller.

14 À Marne-la-Vallée comme à l'Étang-de-Berre, les paysagistes ont été préférés aux architectes pour la conception et la réalisation des espaces publics. Dans l'un et l'autre cas, les choix sont délibérés et sont le fruit d'une collaboration particulièrement étroite, au sein de la maîtrise d'ouvrage, entre ingénieurs et paysagistes. À l'Étang-deBerre, l'ingénieur incite les bureaux d'études techniques à intégrer dans leurs équipes des paysagistes ${ }^{27}$ :

C'est un métier en devenir encore à cette époque-là, le paysagiste... Le métier n'était pas très développé... C'était clair que c'était pas à l'architecte de concevoir les espaces publics [...] on a connu parfois des difficultés avec les architectes.

De même à Marne-la-Vallée, sous la houlette de l'ingénieur, responsable du service des infrastructures, la priorité est donnée aux paysagistes pour les aménagements extérieurs des opérations ${ }^{28}$ :

Le choix des maîtres d'œuvre pour les aménagements extérieurs que l'on faisait c'était à chaque fois un psychodrame pour les (architectes-)urbanistes, c'était souvent contre eux. Les paysagistes étaient préférés aux architectes.

Selon la façon d'organiser le travail, de le diviser, de le recomposer, de développer des compétences en interne ou de faire appel à telle ou telle compétence extérieure, chaque EPAVN a développé son style de maîtrise d'ouvrage. Le domaine et le contenu des tâches de chaque professionnel en découlent, ceci autant que les qualifications supposées ou l'expérience passée des uns et des autres. S'en dégagent aussi des espaces à chaque fois singuliers de confrontation, en interne comme entre commanditaire et prestataire. Les diverses positions des paysagistes dans les configurations professionnelles font alors figure de révélateurs de ces différentes formes d'organisation. Cela étant dit, si ces variations locales mettent en lumière une diversité de styles de maîtrise d'ouvrage, l'objectif ultime de tous les établissements est de faire en sorte de vendre dans de bonnes conditions des charges foncières: il en va de l'économie générale de l'aménagement des villes nouvelles. Or ce facteur, ici non pris en compte, a dû jouer sur l'évolution de ces configurations professionnelles, surtout au cours des années 1980-199029.

18 À la résolution de certains problèmes qui n'avaient pas de réponses immédiates, c'est-àdire des compétences reconnues pour les traiter, les paysagistes ont pris une part active et spécifique. Face à de tels problèmes, les professionnels dans leur ensemble ont installé des relations de coopération qui diffèrent de celles qui sont affichées dans des organigrammes ou celles qui sont alignées sur les termes des contrats de prestation. Ces relations se développent sur fond d'apprentissage et de découverte des différentes manières d'aborder des questions non résolues a priori. Et cette recherche collective met les paysagistes en situation de pouvoir faire reconnaître autre chose que ce qui fait leur mandat traditionnel : «l'habillage ». 
Des opportunités pour les pratiques paysagères : le traitement de l'espace public

Pour les grands ensembles, la répartition des fonctions entre organismes intervenants, la division des tâches et des objets (bâti, voirie, " espaces verts ») ont été au principe de leur conception et de leur réalisation ${ }^{30}$. Dans les villes nouvelles, il ne peut en être de même. La formule de l'équipe intégrée au sein de la maîtrise d'ouvrage provoque des collaborations et des interactions entre compétences. Les limites des qualifications admises se trouvent ainsi repoussées, par exemple pour le traitement des espaces publics et encore davantage pour l'évacuation des eaux pluviales.

La notion d'espace public devient d'usage courant au début des années 1970, avec la critique renforcée du Mouvement moderne et de la doctrine fonctionnaliste. Le développement des sciences sociales, en modifiant les perceptions des réalités sociales et urbaines, nourrit cette critique et vient en appui de nouvelles postures professionnelles ${ }^{31}$. Architectes et paysagistes relisent les grandes pages de l'histoire et de la géographie, reprennent de leurs collègues italiens des analyses de la morphologie urbaine et de leurs collègues anglais les approches pittoresques, ou encore voyagent au loin à la découverte des grands espaces. Il s'agit par suite de dire mieux et de concevoir autrement ce que l'on dénommait auparavant «espaces extérieurs » ou "espaces collectifs ", parfois de renouer avec l'esprit des «espaces libres» du début du $\mathrm{xx}^{\mathrm{e}}$ siècle $^{32}$. Le vocabulaire du projet s'enrichit alors de "mémoire ", de «trames", "séquences » et autres éléments «structurants", manifestant, au-delà des mots choisis, la volonté de s'engager dans une autre façon de penser les rapports du social au spatial, la ville devenant pour le professionnel une occasion de parler en tant qu'intellectuel engagé voire artiste.

21 Sans réduire la force de cette "cohérence idéologique", elle n'explique pas complètement le soin apporté à l'aménagement des espaces publics dans les villes nouvelles ${ }^{33}$. Des considérations plus matérielles sont entrées en ligne de compte. Elles tiennent aux données locales, exigences et/ou contraintes, que les professionnels ont été conduits à intégrer ou à interpréter. Ce qui fait la consistance de leur métier entre alors en jeu. Ainsi l'ampleur des infrastructures à réaliser et la disponibilité de grands terrains inconstructibles se traduisent pour le paysagiste de Saint-Quentin-en-Yvelines en autant d'opportunités ${ }^{34}$ :

Les paysagistes étaient aidés parce qu'il y avait de gros réseaux (et donc) des secteurs inconstructibles (nécessaires au passage de grosses canalisations). D'où l'origine des grands mails de Saint-Quentin: ce sont des couvertures de grands réseaux (mails rectilignes).

Au-delà de telles contraintes qui ont "fabriqué » bien des espaces publics des villes nouvelles, la lecture du territoire de Saint-Quentin-en-Yvelines révèle une série de traces laissées par l'histoire. Le paysagiste développe alors l'idée de récupérer les rigoles et aqueducs souterrains pour modeler l'espace ${ }^{35}$. Bassins, fontaines, canal, étangs et bases de loisirs ont été traités par divers maîtres d'œuvre - artistes, architectes, paysagistes - sans que ces aménagements répondent toujours à une fonction hydrologique à proprement parler, sans que non plus le flâneur puisse en saisir immédiatement le sens. La connaissance approfondie du site en est à la fois clé de lecture et clé du projet.

Un second facteur a joué dans l'importance accordée aux espaces publics : l'inquiétude diffuse d'avoir au final une ville éclatée, semis d'opérations sans relations les unes avec les autres. À l'Étang-de-Berre, l'espace public a été la «clé essentielle » sur laquelle il 
était possible d'agir afin d'assurer une certaine unité à cette ville, clé qui conduit au choix de la "plus grande banalité possible ${ }^{36} »$. Cette inquiétude vient de l'analyse du marché immobilier local et de ses conséquences sur la qualité urbaine et architecturale $^{37}$ :

Pourquoi on a tant investi sur les espaces publics ? Parce que très vite on a constaté, en comparaison avec les villes nouvelles de la région parisienne, qu'on était dans l'incapacité de faire une architecture de qualité au niveau des logements... Dans les villes nouvelles en région parisienne, ils arrivaient à sortir des opérations de logement [...] à faire travailler les architectes avant d'appeler les promoteurs.

24 Au sein de l'EPAVN de l'Étang-de-Berre, le couple formé du paysagiste et de l'ingénieur s'efforce de combiner la recherche de qualité et d'économie générale dans les projets. Le travail s'avère particulièrement fructueux et inventif, passant du diagnostic partagé au calcul précis de la répartition des ressources financières ${ }^{38}$ :

On a inventé de nouveaux systèmes de voirie (avec un ingénieur de l'EPAVN), de nouvelles coupes-types en travers et tout l'argent qu'on économisait sur la manière de gérer les eaux pluviales [...] on le réinvestissait dans les clôtures et dans les plantations.

Pour l'un de ces praticiens, il s'agissait là « d'expérimentation » qui laisse aujourd'hui un « avant-goût d'inachevés ${ }^{39}$ ». En fait, dans nombre de situations à l'Étang-de-Berre comme ailleurs, l'espace public reste un objet tiraillé entre des façons contradictoires de voir et de travailler. D'abord bien des EPAVN se sont vu opposer les normes techniques des Directions départementales de l'Équipement (DDE) (géométrie, gabarit, traitement de la voirie). La hiérarchisation des politiques publiques a fait qu'à Lille-Est le choix fut immédiat et en défaveur des espaces publics de la ville nouvelle: l'autoroute Lisbonne-Stockholm qui traverse la ville impose de longues coupures alors que l'on espérait ici un parc ou là un boulevard urbain. Ensuite, au sein même des établissements, les façons de voir et de faire n'ont pas toujours réussi à s'accorder ${ }^{40}$ :

Ce sont deux approches différentes. Le travail sur le vide donne aux paysagistes leur place: les urbanistes dessinent les plans de masse vus d'avion alors que les paysagistes assurent l'échelle du piéton.

On le voit: si en villes nouvelles les espaces publics ont connu des sorts très variés, les paysagistes ont pu, de temps à autre, exercer leur métier au-delà de «l'habillage ». Les possibilités qui leur étaient offertes ou les occasions qu'ils ont saisies supposaient de leur part de pouvoir prendre pied dans une situation et de prendre langue avec d'autres professionnels. Ici, ce fut en tirant parti des disponibilités foncières, du coût des infrastructures, de l'état du marché immobilier ou de la doctrine d'un EPAVN qui parie sur la continuité du paysage urbain pour réduire les risques que présente une urbanisation par tranches opérationnelles. Là, ce fut à travers le souci d'inscrire les espaces publics dans un territoire qu'il leur fallait lire à la lumière de l'histoire. Là enfin, ce fut en collaborant de façon étroite avec des praticiens aux compétences différentes pour imaginer des usages de l'espace dépassant les logiques sectorielles, qu'elles soient techniques ou économiques.

Des objets complexes révélateurs du noyau des compétences

Dans les EPAVN, les professionnels ont été confrontés à un problème épineux, celui de l'évacuation des eaux pluviales dans des secteurs faiblement urbanisés. Ce problème a trouvé sa solution dans la création, ici ou là, de "bassins d'orage " ou de «lacs artificiels». Davantage que pour l'espace public ou la planification territoriale ${ }^{41}$, le traitement de ce problème passe par les paysagistes. C'est probablement leur apport le 
plus spécifique, et le moins controversé au sein des EPAVN - en dehors de « l'habillage »-, à l'aménagement des villes nouvelles.

Dans les années 1960, sur l'évacuation des eaux pluviales, les compétences sont en France et depuis fort longtemps entre les mains des ingénieurs des Ponts et chaussées dont les formules mathématiques et les abaques ne touchent que les secteurs densément urbanisés, c'est-à-dire largement imperméabilisés (avec la formule dite de Caquot) ${ }^{42}$. Les citadins versent alors leurs eaux, soit dans des égouts existants qui ne sont pas généralisés, soit dans des ruisseaux rapidement transformés en égouts à ciel ouvert. En même temps, les eaux usées mêlées aux eaux pluviales sont encore massivement destinées aux rivières. Dans ces années 1960, si la doctrine des Ponts et chaussées fait force de loi, elle commence à être contestée. Mais les expériences, croisant à la fois les formules de l'ingénieur hydraulicien, de l'ingénieur sanitaire et les observations de l'écologue, c'est-à-dire le calcul des canalisations, les normes de santé publique et les premières préconisations en matière d'environnement sont alors en France fort peu constituées. Elles le sont davantage et depuis plus longtemps en Allemagne et aux Pays-Bas où l'on cherche moins à évacuer qu'à traiter sur place des eaux plus ou moins polluées. Font foi de cette faiblesse française, les tergiversations, débats de doctrine et conflits institutionnels pour rendre "vivante" la rivière Orge (dans le nouveau département de l'Essonne) ${ }^{43}$.

Sur les vastes territoires des villes nouvelles, il a fallu en cette matière expérimenter et en même temps saisir une opportunité. La contrainte socio-économique (coûts d'énormes canalisations avec risques d'inondations en aval) est l'occasion d'explorer des solutions alternatives à un problème posé jusque-là en termes technicoéconomiques, les exigences socio-environnementales étant plus faibles qu'elles ne le sont aujourd'hui. A posteriori, il faut souligner que le choix des bassins d'orage a souvent été fait sans que l'on ait eu à cette époque le souci d'évaluer ce que pouvait représenter la «perte » des charges foncières ainsi extraites de l'économie générale de l'aménagement ${ }^{44}$.

La réalisation des bassins d'orage vient donc du problème que pose la taille des surfaces urbanisées et urbanisables. La démarche préconisée par un bureau d'études néerlandais ayant fait ses preuves sur d'autres sites que les villes nouvelles ${ }^{45}$ consiste d'abord à définir le volume de stockage avec un marnage donné, à en déduire une surface, un profil, un fond de forme puis à reconstituer un milieu biotope pour le développement de la faune. Il s'agit aussi de répondre aux exigences sociales des futurs riverains (nuisances du fait des risques d'odeurs, de moustiques, etc.) Cette solution supposait donc l'intégration de savoirs et savoir-faire distincts et des appuis réciproques entre praticiens.

31 À l'EPAVN de Marne-la-Vallée, les paysagistes conçoivent et dirigent les travaux de nivellement général des parcs et bassins. Ils savent ce qu'il en est de la grande échelle et maîtrisent la topographie. Pour dessiner les bassins dont la position et l'emprise sont décidées par le bureau d'études, ils réalisent une maquette au $1 / 500$, avec toutes les courbes de niveaux ce qui est, pour eux, un «moment fort où le site se révèle avec le bout des doigts - comme les dunes de sable ${ }^{46}$ - ». Le modelé est ensuite reporté sur un plan : «La moindre pente a été expérimentée, ce qui a permis de créer des reliefs très doux. » La priorité pour les paysagistes était « le respect du vallon, des grandes prairies et des couloirs d'eau ». Leur domaine d'expertise s'étend ainsi du site aux différentes dimensions du milieu naturel. 
Cette expérience souligne les connivences entre des paysagistes et des ingénieurs qui sont là plus agronomes que "routiers» ou constructeurs. Elle indique aussi les caractéristiques du métier du paysagiste, au sens le plus artisanal du terme, l'œil et la main venant marquer des différences entre des manières de faire et entre des manières de sentir ${ }^{47}$ :

Il y a une énorme différence entre la façon de travailler des architectes et des paysagistes; l'aménagement des plans d'eau en rend parfaitement compte. À l'époque, les paysagistes travaillaient avec d'immenses maquettes de liège recouvert de plastiline. Les modelés réalisés à la main ont précédé les plans réalisés par la suite. C'est intéressant parce qu'on n'était pas sur des plans qu'on a a priori à petite échelle puis qu'on agrandit progressivement. Là d'entrée de jeu, on travaillait sur de très grandes maquettes et on faisait des modelés. La méthode est intéressante parce que ça ne donne pas le même résultat. En plus, les échelles auxquelles les paysagistes travaillaient n'étaient jamais les mêmes que celles des architectes.

Cette découverte en provoque d'autres, concernant la compétence des uns et des autres, les limites de l'objet sur lequel porte la compétence de chacun, la façon dont celle-ci est maîtrisée, les outils qui sont employés pour travailler. L'échelle de prédilection fait une fois de plus la différence ${ }^{48}$ :

Architectes et paysagistes ne travaillent pas à la même échelle... Les paysagistes faisaient des maquettes des sites, alors que les architectes travaillaient de manière plus abstraite [...] c'est loin d'être négligeable sur la manière dont le paysage a été structuré...

L'approche des diverses dimensions de ce qui fait un site, la prise en compte de ses transformations avec l'importance donnée au temps, comme la mise en forme du « vide » appellent des savoirs et des savoir-faire que ni les architectes, ni les ingénieurs ne peuvent a priori faire valoir. Ces compétences, les paysagistes ont pu les mettre en avant. Ils les puisent largement dans «l'art des jardins » avec ce que celui-ci suppose de connaissances des rapports entre le modelé du terrain, la nature des sols, l'eau, le végétal mais aussi l'ensoleillement. Les paysagistes les traduisent dans des maquettes visibles de tous dans les EPAVN et dont la confection passe aussi pour eux par l'exercice du regard et du toucher.

Des élargissements de compétences et des épreuves

Qu'ils soient au départ ingénieurs, architectes, géomètres, administrateurs ou paysagistes, les professionnels en villes nouvelles ont été conduits à repenser la qualification qui leur était reconnue que ce soit par un diplôme, un titre ou par les représentations que les titres et les diplômes entretiennent. Ces qualifications, les compétences professionnelles qu'elles sont supposées induire, voire les expériences antérieures de ces professionnels ont été dans les EPAVN largement déplacées. Pour les uns ce fut l'occasion d'un retour à la culture générale avec les leçons de l'histoire, de la géographie, de la lecture morphologique ${ }^{49}$. Pour d'autres, ce fut la découverte des effets non désirés des premières réalisations avec par exemple l'observation des décalages entre l'espace public tel qu'il avait été conçu et ses usages effectifs. Pour d'autres encore ce fut la leçon de l'économie de l'aménagement, avec la prise de conscience de la relation entre le salaire de chacun et des bilans annuels des EPAVN. Pour d'autres encore, la définition même de l'aménagement s'en est trouvée modifiée. Ainsi un ingénieur des Ponts rappelle sa surprise en découvrant la multiplicité des relations que la production urbaine oblige à envisager. Son passage en villes nouvelles lui a fait 
toucher une réalité plus complexe que celle qu'il avait connue en DDE, ce qui a attisé sa curiosité ${ }^{50}$ :

Comment on fabrique une ville? Comment on fait? Il faut mettre des voiries, des réseaux, etc. C'est une vision d'ingénieur. Mais l'urbanisme c'est plus que cela.

Ces ouvertures, apprentissages et découvertes ont aussi touché les architectes dont certains disent avoir beaucoup appris des manières de faire des paysagistes ${ }^{51}$. Et tous ont découvert les tenants et aboutissants de la maîtrise d'ouvrage et ont contribué à sa redéfinition. Un paysagiste travaillant dans un EPAVN témoigne, pour ce qui le concerne, du soin particulier qu'il a dû apporter à son travail, en raison à la fois de sa compétence propre et de sa position particulière au sein de son établissement ${ }^{52}$ :

Dans la remise d'ouvrage des accotements de voies et des espaces publics au SCA [Syndicat communautaire d'agglomération], j'apprends mon métier de paysagiste. J'apprends à travailler et à rechercher la qualité. Ils [les services du Syndicat] ont aussi leurs exigences.

Ce faisant, ce travail au sein de la maîtrise d'ouvrage n'est pas sans comporter d'épreuves. Pour les architectes, la tentation a toujours été grande de revenir à un statut de maître d'œuvre, aux savoir-faire initiaux, de rompre ainsi avec une expérience particulière de l'aménagement. Nombreux sont ceux qui, faisant ce choix, garderont cependant un vernis voire une carte de visite "d'urbaniste ». Ce rapport entre pratique de l'architecture et pratique de l'aménagement est une question lancinante. Elle est soulevée en 1968 à Cergy par des urbanistes britanniques qui pointent les défaillances de la culture urbaine (et urbanistique) d'architectes qui se posent en maîtres d'ouvrage alors qu'ils font des propositions de maîtres d'œuvre ${ }^{53}$. Les paysagistes n'ont pas été moins tentés par ce retour à la maîtrise d'œuvre.

38 Au final, le cas de la réalisation des villes nouvelles françaises tend à montrer que le champ des métiers de l'aménagement - tel qu'on peut l'entendre aujourd'hui - s'est constitué non seulement avec l'application des procédures de la planification urbaine mais aussi à travers la gestion de questions floues, c'est-à-dire des objets mal identifiés sur lesquels les prérogatives de tel ou tel métier ne sont pas évidentes ou sur lesquels les expériences passées ne sont pas mécaniquement mobilisables ${ }^{54}$. Des expertises légitimes, autorisées et «mandatées » existaient peu; des opportunités étaient donc à prendre pour les détenteurs de savoirs et savoir-faire restés jusque-là discrets.

Quelles que soient les différences d'organisation, la vocation et le statut des EPAVN ont mis en débat d'anciennes frontières (ainsi celle des grands ensembles entre voirie, bâtiment et espaces verts). Mais ce cadre avait ses propres contraintes: le temps, le territoire et les objectifs politiques et économiques assignés, parfois en haut lieu. Cette gestion de questions floues se lit donc aussi dans les découvertes que provoquent les apprentissages collectifs autour de problèmes concrets que pose l'aménagement de l'espace et qui se révèlent au fur et à mesure, dans l'activité même des professionnels ; les paysagistes, bien que peu nombreux, ont fait des incursions en prenant part ici où là à la résolution de ces problèmes. Reste que, aux dires des professionnels aujourd'hui, bien des occasions d'interaction et de collaboration sont restées inexplorées, par exemple celle qui aurait visé une meilleure lisibilité des villes nouvelles ou celle de la gestion future des équipements et aménagements. 


\section{NOTES}

1. V. Claude, Faire la ville. Les métiers de l'urbanisme au XXe siècle, Marseille, Éditions Parenthèses, 2006, chapitres 3 et 4 .

2. Cette contribution tire parti des recherches menées dans le cadre du Programme interministériel d'histoire et d'évaluation des villes nouvelles françaises (PIHEVN), notamment : V. Claude, J.-C. Fredenucci, Expériences professionnelles et effets de génération en villes nouvelles (1965-2002), 2 vol., Institut d'urbanisme de Lyon, LATTS-ENPC, 2004 ; $\mathrm{N}$. Eleb-Harlé et al., Hydrologie et paysages urbains en ville nouvelle, Laboratoire IPRAUS, École d'architecture de Paris-Belleville, 2004 ; A. Hérat et al., L'espace public en villes nouvelles, Lille, CLERSE-IFRESI (M. Rautenberg et J.-S. Bordreuil pour la direction scientifique), 2004 ; A. Korganow et al., L'équipement socioculturel en ville nouvelle. Les déclinaisons de la formule innovante de l'intégration, Laboratoire ACS, École d'architecture Paris-Malaquais, 2005.

3. V. Claude, « De "l'ensemble"à la ville : flottements des années 1960 et puissance de l'extraterritorialité », revue Histoire urbaine, n 17, déc. 2006, p. 27-46.

4. Par profession, on entend une forme instituée c'est-à-dire capable par divers moyens (diplôme et monopole du titre par exemple) d'être reconnue, de formuler et de faire entendre des intérêts communs, les activités qui en relèvent pouvant être très diverses. C. Dubar, P. Tripier, Sociologie des professions, Paris, A. Colin, 1998.

5. On prolonge ici F. Dubost, «Les nouveaux professionnels de l'aménagement et de l'urbanisme ", Sociologie du travail, 27 (2), 1985, p. 154-164.

6. F. Dubost, « Les paysagistes et l'invention du paysage », Sociologie du travail, vol. 25 (4), 1983, p. 432-445 ; C. Circé, F. Dubost, La profession de paysagiste, Centre de sociologie des arts, EHESS, 1985 (pour la Mission de la recherche urbaine, ministère de l'Équipement) ; thèses en cours d'I. Estienne à l'université de Lille-I, de B. Blanchon à l'École nationale supérieure du paysage de Versailles.

7. «Quels apports des villes nouvelles dans les pratiques professionnelles de l'aménagement aujourd'hui ?» Actes de la Journée d'études du 13 avril 2005 du PIHEVN et du Club Ville et aménagement, ministère des Transports, de l'Équipement, du Tourisme et de la Mer, 2005.

8. R. Castel, «L'expert mandaté et l'expert instituant », Situations d'expertise et socialisation des savoirs, Actes de la Table ronde organisée par le CRESAL à SaintÉtienne les 14-15 mars 1985, CRESAL, 1985.

9. De façon générale, voir les travaux du PIHEVN et de façon plus particulière pour la région parisienne, $\mathrm{L}$. Murard et $\mathrm{F}$. Fourquet, La naissance des villes nouvelles. Anatomie d'une décision (1961-1969), Paris, Presses de l'École nationale des Ponts et Chaussées, 2004 (témoignages réunis en 1975).

10. V. Claude, J.-C. Fredenucci, op. cit., p. 31-36 et rapport annexe.

11. J.-C. Fredenucci, De l'Afrique noire aux villes nouvelles, (thèse en cours à l'Institut d'urbanisme de Lyon).

12. Témoignage d'un ingénieur des Ponts et chaussées, in V. Claude, J.-C. Fredenucci, p. 81 et rapport annexe, op. cit. note 2.

13. A. Hérat, p. 126, op. cit. note 2.

14. Témoignage d'un élu, ibid., p. 51.

15. Témoignage d'un programmateur, in A. Korganow, p. 42, op. cit. note 2. 
16. A. Hérat, p. 163 , op. cit. note 2 .

17. Ibid., p. 143.

18. F. Theulé, « La loi Rocard du 13 juillet 1983 : loi d'aménagement, loi militante ou loi d'élus? ", in L. Vadelorge (dir.), Gouverner les villes nouvelles. Le rôle de l'État et des collectivités locales (1960-2005), Paris, Le Manuscrit, 2005, p. 271-295.

19. Voir les contributions réunies dans «Politiques et marchés du logement dans les villes nouvelles. Quid de la mixité sociale?", Actes de la Journée d'études du 19 avril 2005 du PIHEVN et du Conseil général des Ponts et chaussées, ministère des Transports, de l'Équipement, du Tourisme et de la Mer, 2005.

20. V. Claude, « Les équipes d'aménagement des villes nouvelles. Les avatars d'une expérience collective nationale », Les annales de la recherche urbaine, $n^{\circ} 98,2005$, p. 15-24.

21. V. Claude, J.-C. Fredenucci, rapport annexe, op. cit. note 2.

22. Témoignage d'un responsable de la programmation des équipements, in N. ElebHarlé, p. 95, op. cit. note 2. Il faut relever que des paysagistes maîtres d'œuvre revendiquent aujourd'hui un lien privilégié avec les architectes de EPAVN de Marne-laVallée, ce qui nuance quelque peu cette apparente ligne de démarcation, voir le témoignage de G. Vexlard, Quels apports des villes nouvelles dans les pratiques professionnelles de l'aménagement aujourd'hui ?, p. 26-28, op. cit. note 7.

23. A. Hérat, p. 131, op. cit. note 2. Sur les normes alors en vigueur : « L'équipement pour l'homme ", Urbanisme n 90/91, 1965 et Cahiers de l'IAURP, n² 23, 1971.

24. Témoignage d'un architecte-urbaniste, in V. Claude, J.-C. Fredenucci, p. 77, op. cit. note 2 ; pour les programmateurs, A. Korganow, op. cit. note 2.

25. Témoignage d'un paysagiste maître d'œuvre, in A. Hérat, p. 132-133, op. cit. note 2.

26. Ibid.

27. Témoignage d'un ingénieur, ibid., p. 133.

28. N. Eleb-Harlé, p. 96, op. cit. note 2.

29. On songe par exemple aux conséquences de la prise en compte de la gestion des équipements dans les équilibres financiers plus tendus ou à celles des attentes politiques visant à « réduire les espaces verts » (Voir supra).

30. F. Parfait, « Conception, organisation, réalisation des grands ensembles d'habitations », Urbanisme, n65, 1959, p. 18-39.

31. A. Drouard (dir.), Le développement des sciences sociales en France au tournant des années soixante, Paris, Éditions du CNRS, 1983. Henri Lefebvre a eu une forte influence sur les milieux professionnels de l'aménagement dès 1960-1965, à la fois par ses écrits et par son enseignement à l'université de Nanterre. Plus largement pour les contributions des sociologues et géographes à la critique de l'aménagement urbain des années 1960, Archives nationales F14 770685 Art. 12 et 780633 Art. 4-5.

32. Pour la généalogie de la notion d'espace public : I. Billiard, Les espaces publics, Paris, La Documentation française, 1988 (programme du Plan urbain). Voir les témoignages d'architectes, tels C. Devillers et J. Tribel, in A. Hérat, p. 30 et 34, op. cit. note 2.

33. F. Dubost, « Les nouveaux professionnels de l'aménagement et de l'urbanisme », art. cit.

34. Témoignage d'un paysagiste, in N. Eleb-Harlé, p. 76, op. cit. note 2.

35. Ibid., p. 77.

36. Témoignage d'un ingénieur, directeur de l'EPAVN, et du paysagiste, in A. Hérat, p. 130 , op. cit. note 2 .

37. Ibid., p. 129 et 141 . 
38. Ibid., p. 129.

39. Témoignage d'un ingénieur, ibid., p. 162.

40. Témoignage d'un professionnel (non identifié), in N. Eleb-Harlé, p. 84, op. cit. note 2.

41. Dans les années 1965-1975, à Cergy, l'alliance entre géographes et architectes autour de la planification urbaine, des secteurs à protéger et des directives d'aménagement diffère assez largement de la découverte sensible et quasi anthropologique des jeunes paysagistes de ce qui fait pour eux le " grand paysage ». Cette différence tient autant aux effets de métier, de génération que d'EPAVN. Lire les témoignages de B. Warnier et G. Vexlard, «Quels apports des villes nouvelles dans les pratiques professionnelles de l'aménagement aujourd'hui ? », op. cit. note 7.

42. G. Dupuy, G. Knaebel, Assainir la ville hier et aujourd'hui, Paris, Dunod, 1982.

43. V. Claude, A. Guillerme, L'Orge et ses syndicats. Approche historique d'une rivière périurbaine, École nationale des Travaux publics de l'État, pour le Plan Urbain (MELT), 1986, 156 p. Sur l'antériorité des réalisations allemandes, néerlandaises et pour partie britanniques qui tiennent aux travaux sur les conditions de drainage des eaux usées et d'auto-épuration des rivières, ibid., p. 37-50 et 109-116.

44. Intervention de Michel Rousselot ancien directeur de l'EPAVN de Marne-la-Vallée à la journée : Quels apports des villes nouvelles dans les pratiques professionnelles de l'aménagement aujourd'hui ?, p. 46-47, op. cit. note 7.

45. Le bureau d'études Sauveterre a pour compétence initiale l'agronomie (hydraulique appliquée à l'agriculture, assainissement des eaux usées) mais aussi l'écologie et l'art des jardins. Ses origines et références sont essentiellement hollandaises. Ce bureau d'études dirigé par Erik Jacobsen « avait une sensibilité au paysage et a fait avancer la réflexion : il définissait les zones perméables, les logiques de réseau et les aires à déverser ", in N. Eleb-Harlé, p. 83-93, op. cit. note 2.

46. Témoignage d'une paysagiste, ibid., p. 84.

47. Témoignage d'un professionnel (non identifié), ibid., p. 69.

48. Ibid., p. 103 et 184 .

49. C'est le cas de B. Warnier à Cergy, voir son intervention à la journée Quels apports des villes nouvelles dans les pratiques professionnelles de l'aménagement aujourd'hui ?, op. cit. note 7 .

50. V. Claude, J.-C. Fredenucci, p. 95, op. cit. note 2.

51. Témoignage d'un architecte, in N. Eleb-Harlé, p. 82, op. cit. note 2.

52. V. Claude, J.-C. Fredenucci, p. 113, op. cit. note 2.

53. V. Claude, « De "l'ensemble" à la ville : flottements des années 1960 et puissance de l'extraterritorialité ", art. cit.

54. Sur ce thème de l'urbanisme comme ensemble « flou », voir le chapitre 5 de V. Claude, Faire la ville..., op. cit. note 1.

\section{RÉSUMÉS}

Entre le milieu des années 1960 et le milieu des années 1980, les Établissements publics d'aménagement des villes nouvelles ont été l'occasion pour de jeunes professionnels 
d'expérimenter de nouvelles façons de fabriquer la ville. Architectes, ingénieurs et paysagistes, qui pour la plupart découvrent alors l'aménagement urbain, font valoir leurs savoirs et savoirfaire spécifiques, les enrichissent au sein de maîtrises d'ouvrage fortement structurées et apprennent à travailler en équipe ; ainsi par leurs pratiques, ils ont contribué à redéfinir le champ professionnel dans son ensemble. Deux thèmes ont été particulièrement travaillés par les paysagistes dans leur apprentissage de l'aménagement : celui des espaces publics et celui des bassins d'orage (ou lacs artificiels), thèmes qui font alors problème et sur lesquels ils ont pu montrer qu'ils n'avaient pas pour seule compétence d'« habiller » l'espace.

Viviane Claude, The French new towns as training places for city planning

Between the mid-1960s and the mid-1980s, the public bodies involved in planning French new towns provided young professional planners with the opportunity to try out new ways of "creating" cities. Architects, engineers and landscapers, most of whom were taking their first steps in city planning, were able to develop and enrich their specific knowledge and expertise through highly structured project management and to learn to work as part of a team. Thence, they helped redefine the entire professional environment. Landscapers focused on two problematic themes in particular in the course of their city planning apprenticeship - public spaces and storm basins (or artificial lakes) - thus allowing them to show that they were more than simply outdoor "window dressers".

\section{INDEX}

Mots-clés : paysage urbain, villes nouvelles françaises, apprentissage, urbanistes, paysagistes Keywords : urban landscape, french new towns, apprenticeship, city planners, landscapers

\section{AUTEUR}

\section{VIVIANE CLAUDE}

Historienne de l'urbanisme, chercheur au LATTS, École nationale des Ponts et chaussées, 6-8 avenue Blaise-Pascal - Cité-Descartes 77455 Marne-la-Vallée Cedex 2, viviane.claude@univ-lyon2.fr 